\title{
Memoria e identidad: la obra del escultor alemán Ludger Hinse
}

\author{
Memory and identity: the work of German sculptor \\ Ludger Hinse
}

\author{
Natalia TIELVE GARCÍA \\ Universidad de Oviedo
}

Recibido: 13-IV-2015 / Aceptado: 13-V-2015

RESUMEN: En este trabajo se aborda, en su versatilidad y diversidad plástica, la obra del creador Ludger Hinse. Nacido en Recklinghausen, en 1948, forma parte de una generación de artistas alemanes crecidos en la postguerra, circunstancia que ha marcado en amplio grado el devenir de su trayectoria vital y profesional. Buena parte de sus propuestas responden a una visión arqueológica donde la memoria del trabajo y la memoria del lugar ocupan un puesto destacado. $\mathrm{Al}$ amparo de estas premisas, ha ido entretejiendo un discurso que ahonda en la interacción arte e industria y, de forma más específica, en la relación entre la escultura y la arqueología industrial.

Palabras clave: Ludger Hinse, Arte, Técnica, Acción Creativa, Memoria.

ABSTRACT: In this paper we study, both in its versatility and its plastic diversity, the work of creator Ludger Hinse. Born in Recklinghausen in 1948, he is part of a generation of German artists raised in the postwar years, circumstance that has greatly influenced the progression of his vital and professional pathways. Many of his proposals correspond to an archaeological vision in which the memories of labor and place hold a noted position. Under cover of these premises, the artist has gradually woven a discourse that delves into the interaction between art and industry and, in a more specific way, into the relationship between sculpture and industrial archaeology.

Keys words:Ludger Hinse, Art, Technique, Creative Actions, Memory.

\section{LUDGER HINSE: UNA POLIÉDRICA TRAYECTORIA}

Nacido en Recklinghausen, en 1948, Ludger Hinse forma parte de una generación de artistas alemanes crecidos en la postguerra, circunstancia que ha marcado en buena medida el devenir de su trayectoria vital y pro- fesional ${ }^{1}$. Su infancia y juventud en el enclave industrial del Ruhr, su actividad pública, con una notoria proyección en el ámbito de lo sin-

${ }^{1}$ Este trabajo se adscribe al Proyecto de Investigació. Espacios portuarios y villas costeras: modelos de estrategias urbanísticas y patrimoniales en regeneración y transformación del litoral asturiano (HAR2011-24464), auspiciado por el Ministerio de Ciencia e Innovación. 
dical y lo político, el impacto generado por el desmantelamiento de la industria y los planes de reconversión y de regeneración socioeconómica desarrollados en las últimas décadas, han sustentado conceptual y matéricamente su trabajo. No en vano en su obra puede advertirse un decidido proceso de identificación con el entorno. Procedente de una humilde familia de tradición minera, formado en la Escuela de Trabajo Social de Bochum entre los años 1968 y 1972, ejerció como secretario general de los trabajadores metalúrgicos de la Cuenca del Ruhr, situándose al frente del IG Metall en Bochum. Desde los años 1970 ha compaginado y entrelazado su actividad pública con una inquietud artística sin fisuras, que le ha llevado a discurrir por los territorios de la escultura, la pintura y la acción creativa. Cabe subrayar la proyección internacional que los trabajos del alemán han alcanzado, dándose a conocer, entre otros espacios, en el Instituto Goethe de París, el Museo de Arte Contemporáneo de Santiago de Chile, el Lamdesmuseum de Emden, la Galería Chamissoplatz de Berlín, la UNEAC de La Habana, el Grochtmann-Museum de Datteln, la Galería Rath de Colonia, la Galería Open Art de Borken, el Altes Schloss de Dillingen, o, en el caso de nuestro país, el Museo Juan Barjola de Gijón².

En su discurrir profesional Hinse ha rastreado cauces de expresión diversos, acudiendo a recursos plásticos y visuales que van desde la disciplina pictórica a la indagación escultórica, sin eludir la vía performática, en un incesante afán de crear y de experimentar. En todo caso, con independencia del medio, de los materiales o de las formas que definen sus propuestas, su trabajo en conjunto responde a una evidente coherencia. Hay en sus obras una razón de ser común que las anima $\mathrm{y}$, ante todo, una coherencia de propósito: la convicción de que el arte puede suscitar un estado de reflexión y propiciar una intervención sobre el entorno. Sus inquietudes políticas, en este sentido, al igual que su talante ex-

\footnotetext{
${ }^{2}$ Acerca de la exposición de sus trabajos en el Museo Juan Barjola de Gijón puede consultarse: N. TIELVE, Ludger Hinse. Encrucijada o trasmutación de los valores, Oviedo, 2001.
}

trovertido y sus dotes de buen comunicador, han sido catalizadores vitales de su labor.

Retrospectivamente, podemos distinguir en su producción un impulso hacia el rigor conceptual y la depuración formal. En efecto, a lo largo de los años, el artista ha avanzado en la configuración de un lenguaje propio que, superando lo circunstancial, haciendo uso de un código privativo, ha ido ganando en coherencia constructiva, limpieza, simplicidad y vocación ascética. En sus primeros trabajos, durante los años setenta y ochenta, al igual que otros creadores alemanes y europeos, Hinse sintió la necesidad de reflexionar sobre el sentido de la pintura, sobre la práctica del oficio y su papel en el marco de la creación contemporánea. Gestos impulsivos, palpitantes trazos de color de acentuada intensidad y ciertos componentes sígnicos caracterizaban su producción más temprana. Se trata de obras de formato abultado insertas dentro de una tendencia neoexpresionista, inspirada en amplio grado en la tradición expresionista del arte alemán largamente cultivada en aquellos años, en el panorama artístico germano, por creadores como Georg Baselitz, Markus Lüpertz, Karl Horst Hödicke, Anselm Kiefer o Jörg Immendorff ${ }^{3}$ (Fig. 1).

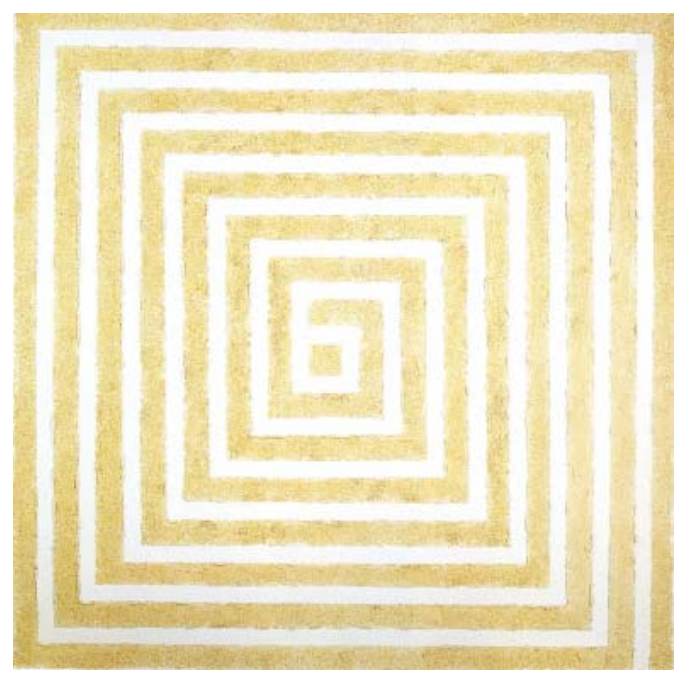

- Fig. 1. S/T. 1995. Oleo sobre plancha de mármol. 160 x $160 \mathrm{~cm}$. Ludger Hinse. Catálogo de la exposición Transparenzen, Museum Bochum.

\footnotetext{
${ }^{3}$ Véase: Geronnenes Feuer, Berlín, 1991; H. GREBING,
} Tränen waren Partner der Träume, Bochum, 1992. 
La gestualidad y el impulso cromático dieron paso, a medida que avanzaba la década de los noventa, a un conjunto de trabajos de mayor contención expresiva, más silenciosos y severos desde el punto de vista formal: despojados de referencias anecdóticas, parcos en el trazo y contenidos en el color hasta alcanzar prácticamente la monocromía del blanco ${ }^{4}$. Lo visible se veía reducido a su expresión mínima, encerrando de alguna manera a cada obra en su propio aislamiento. Hinse reemplazaba la pincelada violenta por sutiles veladuras y por una estructuración compositiva de desnuda geometría y, no obstante, íntima voluntad poética. Gestadas a partir de signos breves, apenas sugerencias de trazos, leves retículas y puntos, las piezas, frágiles y delicadas eran construidas mediante leves transparencias de armónica serenidad. Alcanzaban, sin embargo, una fuerza inusual gracias a los juegos de equilibrios introducidos por el artista.

Paralelamente, en los años noventa, Ludger Hinse se adentraba en la práctica escultórica. Constituye éste el territorio en el que de modo preferente ha proyectado desde entonces su hacer creativo, avanzando en la consecución de un orden acomodado en la tenacidad y no exento de lo que cabría definir como "gesto poético". Desde una perspectiva que podríamos considerar arqueológica, una notoria faceta de su labor se ha basado en la memoria histórica de lo desaparecido, de la actividad fabril y, en especial, de las dinámicas introducidas por la reconversión económica y la crisis del sector industrial, con unas consecuencias inmediatas para su entorno vivencial, la Cuenca del Ruhr. En el marco de esta visión arqueológica-industrial, partiendo de pautas autorreferenciales que giran en torno a su propia experiencia personal, la industria del acero, en lo material y en lo semántico, se vincula estrechamente a sus creaciones. Aunque se ha servido de diferentes materiales, ciertamente, resulta especialmente des-

\footnotetext{
${ }^{4}$ Estimamos de utilidad la consulta de los catálogos: Transparenzen, Ludger Hinse, Bochum, 1998; Die Farbe Weiss. Bewegte Kunts - The color white. Moving Art, Münster, 2002
}

tacable su experiencia con el acero ${ }^{5}$. Materia recurrente, éste es valorado por Hinse no sólo por su potencial expresivo, sino también por su valor simbólico en relación con su propia experiencia vital. Funciona de este modo como elemento connotativo, en términos de identificación cultural, de la memoria del trabajo y de la memoria del lugar ${ }^{6}$ (Fig. 2).

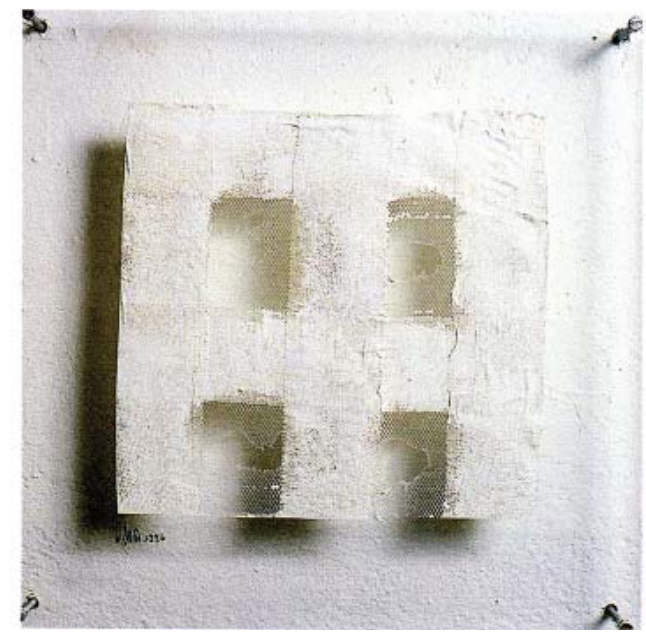

- Fig. 2. S/T. 1996. Collage. Tela metálica, muselina, plexiglás. $26 \times 25 \mathrm{~cm}$. Ludger Hinse. Catálogo de la exposición Transparenzen, Museum Bochum.

Una de las líneas de investigación más interesantes desarrolladas por Ludger Hinse en el campo escultórico ha tenido como objeto de indagación el vacío, tanto desde el punto de vista físico, como conceptual. Así, un grupo importante de sus propuestas pueden entenderse como fruto de la confrontación de elementos opuestos, tales como ausencia/presencia, lleno/vacío y hueco/opacidad, inscrita dentro de una dialéctica de lo ausente. De este modo, a través de lo presente o visible se pone de relieve lo no-presente, lo que queda oculto e insinuado; a través de lo físico -esto es, lo material, tangible y visible- se brinda el acceso a lo no-físico, por consiguiente, espiritual e inmaterial. En lo que atañe a la reflexión sobre las relaciones de la forma con el lugar y en cuanto a la for-

\footnotetext{
${ }^{5}$ Véase: Licht des Stahls, Catálogo de la Exposición celebrada en Werkshatlle 29, Krefeld, 2002.

${ }^{6}$ Véase: J. LOSKILL, Ludger Hinse, Essen, 2013.
} 
malización y a la experiencia del espacio, sus trabajos pueden conectarse con el legado postminimalista. Alejadas por su emocionalidad e intensidad del vacío propio del minimal, sus piezas responden a la búsqueda de una nueva y directa relación entre la obra y el ambiente, donde el espacio se entiende como algo no disociado de la forma. Así, bajo la premisa de que el arte es una forma de transmisión de pulsiones, Hinse ha ido trazando juegos de relaciones, en el plano formal y discursivo, a distintos niveles: una malla de reciprocidades y significaciones provisorias que se abre a múltiples lecturas ${ }^{7}$.

\section{ARTE Y TÉCNICA: UNA ACTITUD DE COMPROMISO ESTÉTICO Y SO- CIAL}

Son numerosos los proyectos de investigación, publicaciones, líneas de trabajo y creaciones artísticas que han ahondado en los últimos años en las relaciones entre el ámbito de lo científico y de lo tecnológico y el arte ${ }^{8}$. En este escenario, desentrañar

7 J. Jiménez en alusión a lo que él mismo define como horizonte privilegiado del arte se refiere a la experiencia estética como una forma de éxtasis: una "experiencia irrepetible, intensa que consigue hacernos olvidar que somos tránsito, fugacidad, que nos permite vivir, sólo por un instante: el instante de plenitud estética, fuera del tiempo, más allá de su pinchazo implacable". En J. JIMÉNEZ , "Arte y Tiempo. Perspectivas en el final de siglo", en R. M. RAVERA (comp.), Estética y Crítica. Los signos del arte, Buenos Aires, 1998, p.32

8 Podemos destacar en esta línea los numerosos trabajos que en los últimos años viene publicando The MIT Press, editorial afiliada al Instituto Tecnológico de Massachusetts, que cuenta con uno de los centros de investigación más activos en este campo, el Center for Art, Science \& Technology, junto con el Center for biological arts de la Aalto University en Finlandia, la Slade School de Londres, la International Society for Arts, Science and Technology de París, o el INBA de México vinculado a las iniciativas de investigación y producción del centro de Arte, Ciencia y Tecnología de la UNAM; todas ellas instituciones basadas en el trabajo interdisciplinar entre artistas y científicos. Entre otras publicaciones pueden refererenciarse: S. EDE, Art and Science, London-New York, 2005; R. ASCOTT, Art, Technology and Consciousness, Bristol, 2000; Ph. HUSBANDS, O. HOLLAND y M. WHEELER, The Mechanical Mind in History. Bos- la obra de Ludger Hinse en toda su dimensión formal y conceptual conduce ineludiblemente a considerar una parte esencial de su producción, la que viene desarrollando desde los años noventa, como fruto del binomio arte-técnica ${ }^{9}$. Se trata de una cuestión que no ha resultado ajena, como es sabido, al mundo de la creación y del discurso artístico a lo largo de la historia. En su conocida obra La mecanización toma el mando Sigfried Giedion subraya el papel predominante que la mecánica ha tenido históricamente sobre el ser humano $\mathrm{y}$, de forma particular, a partir del siglo XVIII de la mano de los logros tecnológicos vinculados a la revolución industrial ${ }^{10}$. Mecani-

ton, 2008; M. LYNCH. y S. WOLGAR, Representation in Scientific Practice. Cambridge, 1990; S. WILSON, Art + Science Now. How scientific research and technological innovation are becoming key to $21^{\text {st }}$ century aesthetics, New York, 2010; B. LATOUR, Nunca fuimos modernos. Ensayo de antropología simétrica, Buenos Aires, 2007; M. FOUCAULT, Tecnologías del yo, Barcelona, 1990; G. CELANT y G. MARIANELLO. Vértigo: A century of multimedia art, from futurism to the web, Milán, 2007; A. MILLER, Colliding Worlds: How Cutting-Edge Science is redefining contemporary, New York, 2014. En el caso de nuestro país: R. PARÉS, “La problemática de la producción artística en el sistema, Arte, Ciencia y Tecnología en el Estado Español", en Informes preparatorios para el Libro Blanco Arte-Ciencia-Tecnología, Madrid, 2012; J. L. BREA, La era postmedia. Acción comunicativa, prácticas (post) artísticas y dispositivos neomedievales, Salamanca, 2002; M. A. GARCÍA et al., Arte y pensamiento en la era tecnológica, Bilbao, 2004; C. GIANETTI, El discreto encanto de la tecnología. Artes en España, Badajoz, 2008.

${ }^{9}$ Discernir en términos de oposición arte y técnica carece de sentido dado que, siguiendo a Pierre Francastel, es en la técnica en donde las artes se emplazan junto con el resto de las actividades específicas del hombre: "mediante el arte, las sociedades hacen el mundo un poco más cómodo o algo más poderoso, y a veces consiguen sustraerlo a las férreas reglas de la materia, o a las leyes sociales y divinas, para hacerlo momentáneamente un poco más humano". En P. FRANCASTEL, Arte $y$ Técnica en los siglos XIX y XX, Madrid, 1990, p.14.

${ }^{10}$ La relación entre la arquitectura, diseño y artes visuales resulta particularmente sugerente en el caso de los creadores que siguiendo la estela de Walter Gropius están ligados al Movimiento Moderno. Formado en la Escuela de Viena y en buena medida seguidor de algunas de las tesis formuladas por Jakob Burckhardt y Heinrich Wölfflin, Sigfried Giedion no dudó en estimar 
zación, racionalidad, gestión científica, estandarización, progreso, convertibilidad, adaptabilidad o economía, por consiguiente, son algunas de las nociones que han repercutido en el entorno del hombre, en las formas de vida y de pensamiento, así como en la práctica de las artes. En el seno de las tendencias contemporáneas, la influencia y frutos más notables de la mecanización pueden percibirse en campos como la arquitectura, el diseño y en general en las artes plásticas vinculadas a lo industrial dentro del discurso del Movimiento Moderno. No obstante, cabría discernir, siguiendo a Gillo Dorfles, un primer periodo paralelo en su discurrir temporal a la primera revolución industrial definido por la distinción entre las consideradas Bellas Artes y las artes aplicadas, entendidas éstas en una dimensión preeminentemente técnica y mecánica, que a lo sumo podían incorporar elementos decorativos que les revestían de un halo de esteticidad. A este período seguiría el del Art Nouveau, íntimamente ligado en sus raíces a la tradición de las Arts \& Crafts, caracterizado por dar cabida a objetos de producción mecánica que reuniesen un determinado componente artístico. En esta fase se imbricaron tanto De Stijl como Bauhaus, movimientos que apostaron con rotundidad por la producción de objetos industriales en el seno del binomio utilidad-belleza y por la unidad plástica de las artes, de la industria y de la técnica ${ }^{11}$. Fue especialmente con Walter Gropius en la Bauhaus de Weimar cuando se planteó abiertamente, con racionalidad germánica, el objetivo de fusionar arte e industria, actuando, eso sí, la

la cultura del progreso, la técnica y la máquina como valores intrínsecos a la sociedad contemporánea, así como los productos creados por el hombre, entre ellos los artísticos: los productos de la técnica tendrían como misión primordial mejorar, hacer más comprensible, más eficiente y más agradable la existencia cotidiana. En S. GIEDION, La mecanización toma el mando, Barcelona, 1978, pp. 490-515.

${ }^{11}$ En G. DORFLES, El diseño industrial y su estética, Barcelona, 1977, p. 37-38. arquitectura como agente mediador ${ }^{12}$. La estética maquinista y el espíritu industrial, alimentados por un pensamiento racional basado en la eficacia del principio de funcionamiento, estimularon por consiguiente la asociación entre arte y técnica como una nueva unidad ${ }^{13}$.

En adelante, dentro de lo que podemos considerar como la industria del arte se han venido introduciendo cambios notables que, en amplio grado, han de ponerse en relación con las conquistas de la técnica moderna, en el terreno de los materiales, de los procedimientos, de las sensibilidades y de los planteamientos teóricos sobre el concepto mismo de arte: el post-arte o el arte postaurático ${ }^{14}$. Las múltiples posibilidades de reproductibilidad mecánica e industrial han establecido nuevas relaciones entre las artes y las actividades técnicas. Por consiguiente, el discurrir de la práctica artística contemporánea, así como el pensamiento teórico gestado a su amparo, ha venido reflejando de una forma directa o indirecta los hallazgos tecnológicos y los avances científicos, en

${ }^{12}$ En S. GIEDION, Espacio, tiempo y arquitectura. Madrid, 1979, p. 513.

${ }^{13}$ Conviene, no obstante, recordar la apasionada defensa de la modernidad que los futuristas sostuvieron: su exaltación de la tecnología, de la máquina, de la velocidad y de la nueva experiencia del tiempo y del espacio propios del universo moderno.

${ }^{14}$ Parece obvia la alusión a la reflexión de Walter Benjamin acerca de la obra de arte en la época de la nueva técnica, a cuyo entender entre las funciones sociales del arte "la más importante es la de establecer un equilibrio entre el hombre y el sistema de aparatos". En W. BENJAMIN, La obra de arte en la época de su reproductibilidad técnica, México, 2003, p. 84. Se trata, en todo caso, de una dinámica que cabría relacionar con la disolución del concepto de autor entendido a la manera tradicional y, en consecuencia, con la desaparición de la lectura unívoca de la obra de arte. Resultan particularmente significativos en este orden de cosas los conocidos textos de Roland Barthes, Michel Foucault y Umberto Eco: R. BARTHES, "La muerte del autor", en El susurro del lenguaje. Más allá de la palabra y la escritura, Barcelona, 1987; M. FOUCAULT, “¿Qué es un autor?”, Litoral, 25/26, 1998, pp. 51-82; U. ECO, "Entre el autor y el texto", Interpretación y sobreinterpretación, Madrid, 2002, pp.80-103. 
campos como la automatización o, más recientemente, la informática y la cibernética ${ }^{15}$ (Fig. 3).

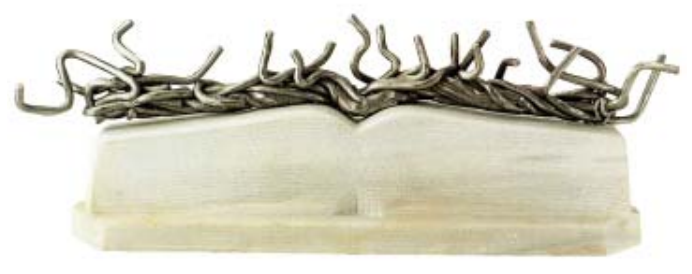

- Fig. 3. Iesende Gefühle. 2001. Acero inoxidable, mármol. $60 \times 26 \mathrm{~cm}$. Ludger Hinse. Catálogo de la exposición Die Farbe Weiss- Bewegte Kunst, BASF Coatings AG.

En este orden de cosas, el trabajo de Ludger Hinse responde a una profunda reflexión estética y a una actitud comprometida con su entorno. Más arriba hemos apuntado al impacto que en sus creaciones ha tenido su experiencia vital: los años de infancia y juventud en la cuenca mineroindustrial del Ruhr, su actividad pública en el ámbito de lo sindical y, singularmente, el conocimiento directo del desmantelamiento industrial, los planes de reconversión y sus amargas consecuencias. La constatación y consciencia de estos fenómenos han guiado una parte importante de su labor, la que le ha permitido ahondar en la interacción arte e industria $y$, más específicamente, en la relación entre la escultura y la arqueología industrial. Resulta oportuno en este sentido subrayar la destacable labor de investigación y de divulgación que el Deutsches BergbauMuseum -Museo Alemán de la Minería en Bochum- ha realizado. La institución, ade-

\footnotetext{
${ }^{15}$ Los territorios del Net-art, el Arte Robótico, el Space Art, el Satellital Art, el Arte Electrónico, la Arquitectura Digital, el Live Cinema, entre otros, ponen de relieve esta acentuada interrelación entre el arte y el ámbito tecno-científico que tan presente está en nuestros días.
}

más de promover una encomiable tarea de indagación y conocimiento de los testimonios del pasado industrial de la Cuenca del Ruhr en particular y del territorio alemán en general, desarrolla una sustancial actividad de exposición y difusión de obras artísticas que tienen como referente el mundo de la industria: pinturas, esculturas, fotografías y grabados de creadores alemanes de distintos períodos y tendencias, en muchas ocasiones de gran valor no sólo artístico sino también documental ${ }^{16}$.

En el marco de este conjunto de actuaciones que promueven el diálogo entre el arte y la industria, Hinse ha colaborado en repetidas ocasiones con el Museo de Bochum desde que, en 1989, la institución organizara la primera exposición de sus trabajos. Con idéntica orientación, en los últimos años ha participado en diversas experiencias que han tenido como escenario espacios industriales obsoletos ${ }^{17}$. Se trata de acciones que han estado dirigidas no solamente a preservar y valorar el interés de estas instalaciones y lugares desde la perspectiva del patrimonio industrial, sino también a cualificarlos

${ }^{16}$ El Museo de Bochum no es la única institución alemana que promueve iniciativas en este campo. Antes bien, a su lado podemos destacar la labor del Westfälisches Industriemuseum y el Deutsche Arbeitsschutz Ausstellung, en Dormund; la Städtische Kunsthalle de Recklinghausen, la Städtische Galerie Schlo Oberhausen y el Rheinisches Industriemuseum, en Oberhausen; el Lehmbruck Museum en Duisburg; el Kustmuseum, en Düsseldorf; o el Ruhrland Museum, el Folkwang y la Villa Hügel, en Essen. La mayor parte de estas instituciones se sitúan en la Cuenca del Ruhr, una de las zonas de más activa industrialización de Europa y, actualmente, uno de los focos sin duda de mayor interés en lo que al Patrimonio Industrial se refiere.

17 Entre otros, el Atelierschiff UNARTIG de Frankfurt (1996), el Gelsenwasser Info-Zentrum de Haltern (2000), la Maschinenhalle Zeche Scherlebeck de Herten (2003) o la Maschinenhalle Friedlicher Nachbar de Bochum (2008). En la misma línea se sitúa su colaboración en el marco del proyecto Kunst in der Fabrik, desarrollado en Duisburg en el año 1996, entre otras experiencias que ha realizado ahondando en la relación arte e industria. 
desde el punto de vista estético ${ }^{18}$ : a través de la práctica creativa el arte, en tanto que técnica espaciadora, permite descubrir, resituar y resignificar estos espacios ${ }^{19}$. Siguiendo estas premisas, la mirada del escultor alemán se ha dirigido frecuentemente a los testimonios de la actividad industrial y especialmente a la vinculada a la producción del acero. No en vano éste ha sido uno de los materiales industriales, junto con el hormigón armado, más ampliamente valorado en términos simbólicos en relación con el avance tecno-científico, con las ideas de cambio, innovación y progreso. El acero, apreciado en su verdad, en su crudeza y en su fuerza plástica, se ha convertido no sólo en el sustento matérico de buena parte de las esculturas de Ludger Hinse sino también en su soporte conceptual en términos de identificación cultural. Los recursos de oficio no resultan ajenos a su producción; antes bien, les han servido como base y guía para proceder a indagar en la materia, para evidenciar la huella del trabajo y el proceso técnico mismo. La impronta del trabajo fabril, en diálogo con el trabajo creativo del artista, se pone de relieve en sus propuestas, evocando, en clave poética y discursiva, a modo de metáfora visual, el proceso industrial seriado. Así, la práctica de la reiteración constituye el sistema operativo que condiciona una parte importante de su labor (Fig. 4).

${ }^{18}$ Se trata de iniciativas que pueden conectarse con una política más amplia de reutilización de espacios históricos que reúnen un determinado valor simbólico para la comunidad, favoreciendo, ante el ciudadano, la memorización afectiva del ambiente y del territorio. Véase G. DORFLES, Del significado a las opciones, Barcelona, 1975, p.195. Con relación a este tipo de experiencias que fomentan el diálogo entre arte e industria se puede consultar: L. BADOSA, "Presencia del arte en el futuro museo industrial", FABRIKART, no 1, 2001, p. 29.

${ }^{19}$ Es la techné la que propicia la apertura, o roza -en relación con el concepto alemán räumen- del espacio: a través del arte el hombre genera espacio, lo ordena y lo transforma, física y mentalmente. A través de la creatividad, en consecuencia, es posible "resignificar" territorios, cuerpos y espacialidades. En F. DUQUE, Arte público y espacio político, Madrid, 2001, p.12.

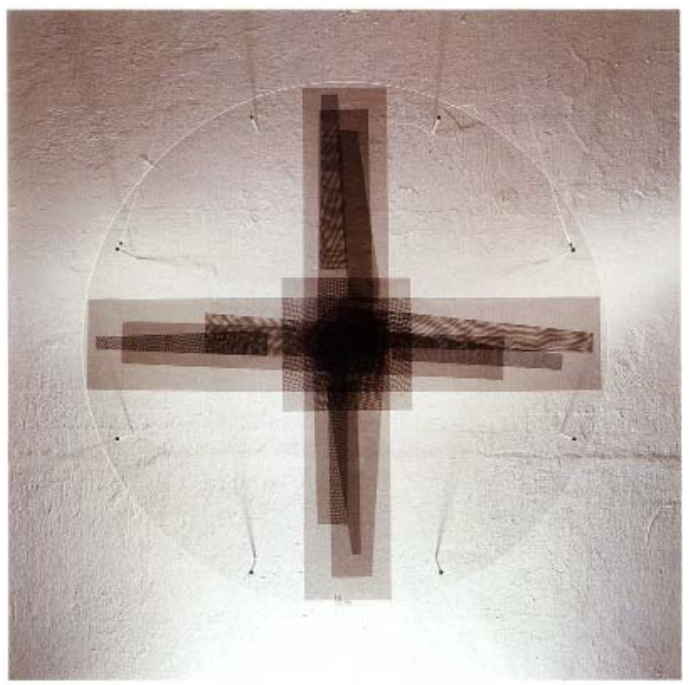

- Fig.4. Lichkreuz 3. 2001. Acero inoxidable, plexigás. 100 x 100 cm. Ludger Hinse. Catálogo de la exposición Ludger Hinse, Museo Barjola.

Asimismo, la búsqueda de un orden semántico se refleja en la disposición metódica y racionalista con la que Hinse acomete sus obras. En una exploración donde el concepto adquiere idéntica importancia que la ejecución, el compromiso, la fuerza y el rigor conviven con el sentimiento y la sutilidad. Son aspectos que, de modo particular, podemos advertir en un nutrido conjunto de trabajos donde prevalecen el linealismo y el pulso etéreo: líneas trazadas en el espacio, a modo de dibujos, con frecuencia retorcidas, que, pese a estar desprovistas de una forma claramente definida, tienen un potencial evocador. Dentro de una suerte de gramática de la repetición, con la contundencia de una reproducción controlada, a modo de cita, establecen analogías y evocan, imitan en cierto sentido, formas, acciones y elementos propios del mundo del trabajo industrial.

\section{ARQUEOLOGÍA DE LA MEMORIA}

Analizando con perspectiva la labor de Ludger Hinse resulta pertinente destacar el interés que ha mostrado por abordar, en un ejercicio de introspección, las nociones de memoria y de identidad. Partiendo de lo íntimo y de lo personal, en clave arqueológica, el artista ha rescatado retazos de historia propiciando, 
en el orden simbólico, una labor de reconocimiento e identificación ${ }^{20}$. Es precisamente lo que sucede en Fünf Tore ${ }^{21}$ realizada con motivo de la declaración del Ruhr como Capital Europea de la Cultura en 2010, dentro de una campaña que integraba cincuenta y tres municipios encabezados por Essen, y que fue desarrollada bajo la dirección artística de Katja Abmann $^{22}$. Explorando las nociones de lugar y de territorio, para acometer este proyecto, Hinse escarbaba en las bases de su memoria personal para devolverla como colectiva ${ }^{23}$ (Fig. 5).

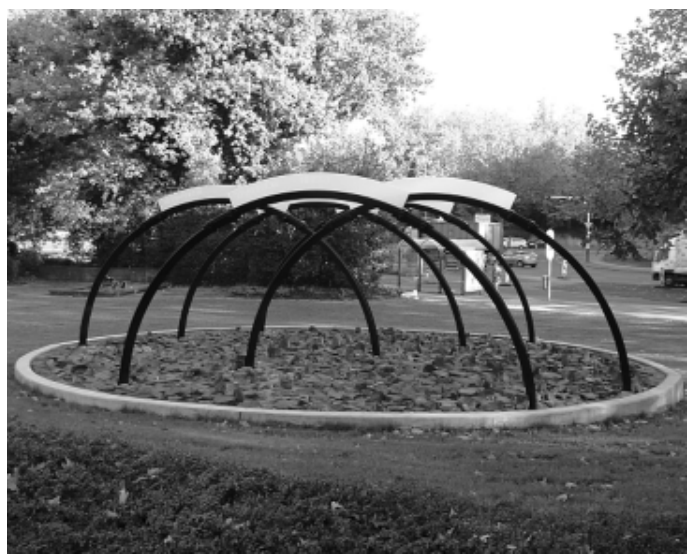

- Fig.5. Fünf Tore. 2010. Acero, vidrio y circuitos de luz. Ludger Hinse. Catálogo de la exposición Emscherkunst 2013, Oberhausen.

${ }^{20}$ Acerca de estas nociones es recomendable la lectura de: J. LE GOFF, El orden de la memoria. El tiempo como imaginario, Barcelona, 1991; F. CHOAY, Alegoría del Patrimonio, Barcelona, 2007; M. CERTEAU, La cultura en plural, Buenos Aries, 1999.

${ }^{21}$ Podemos traducir el título de esta pieza como Cinco Puertas.

${ }^{22}$ A este respecto puede consultarse: F. MATZNER, K.. H. PETZINKA y J. STEMPLEWSKI, Emscherkunst.2010, An Island for the Arts, Ostflildern, 2010; W. SYKORRA, Borbecker halblang. Ein Schulprojekt der Kulturhauptstadt Europas Ruhr.2010, Essen, 2011.

${ }^{23}$ En su dimensión colectiva la memoria puede entenderse como social y política. Resulta interesante acudir en este sentido a la reflexión de Maurice Halbwach sobre la memoria colectiva en tanto que fenómeno sociológico que se genera a través de "flujos de pensamiento continuo, de una continuidad que no tiene nada de artificial puesto que retiene del pasado lo que aún está vivo o es capaz de vivir en la conciencia del grupo que la mantiene". En M. HALBWACH, A memoria coletiva, Sao Paulo, 2006, p. 102.
Fünf Tore, compuesta por cinco arcos de acero entrelazados, que alcanzan los doce metros de altura, vidrio y circuitos de luz, con un peso aproximado de 1,5 toneladas, fue planteada como un paisaje lumínico que reunía un doble sentido: homenaje y denuncia ${ }^{24}$. Utilizando la metáfora de la luz y su propia evidencia física la obra permitía "iluminar" 25 , en el sentido de dar visibilidad al proceso de desindustrialización, rememorando la historia industrial y los conflictos laborales que han marcado el devenir del Ruhr y, por consiguiente, el discurrir vital del artista $^{26}$. Conviene tener presente, en este sentido, el indudable interés patrimonial de la Cuenca del Ruhr, una región que ha hecho de la cultura industrial su razón de $\operatorname{ser}^{27}$.

${ }^{24}$ Fünf Tore, instalada en Bochum, fue construida utilizando los denominados arcos Heintzmann, producidos por la firma del mismo nombre, ampliamente utilizados en la construcción de obra civil, en particular túneles, y también en la minería. Su utilización, como no podía ser de otro modo, respondía a la voluntad de simbolizar la memoria, de mantener viva la llama de tradición. El vidrio, los cristales de colores LISA y los circuitos LED integrados se encargan físicamente de arrojar luz sobre la historia que la pieza rememora.

${ }^{25}$ A propósito de la estrategia de "iluminación" resulta oportuna la lectura de: A. MOLES y R. CAUDE, Creatividad y métodos de innovación, Madrid, 1977, pp. 40-42.

${ }^{26} \mathrm{Al}$ hilo de estas consideraciones parece oportuno recordar la conocida afirmación de Walter Benjamin. “el que abarque con la vista el patrimonio cultural tiene por doquier una procedencia en la que no puede pasar sin espanto. No sólo debe su existencia a los grandes genios que lo han creado, sino también al vasallaje anónimo de sus contemporáneos. No existe un documento de la cultura que no sea a la vez de barbarie". En W. BENJAMIN, La dialéctica en suspenso, fragmentos sobre historia, Santiago de Chile, 1995, pp. 51-52.

${ }^{27}$ Situada en el área de influencia del gobierno regional de Renania del Norte-Westfalia, la Cuenca del Ruhr fue una de las regiones de mayor concentración urbana e industrial de Europa, aunque también una de las más duramente afectadas por la crisis y el desmantelamiento industrial -particularmente en los sectores de la minería del carbón y de la siderurgia- desde los años setenta, que dieron como resultado un paisaje desolador que reflejaba la profunda depresión económica de la zona. El estado alemán, para tratar de hacer frente a la situación, ideó un proyecto de gran envergadura, modélico, dirigido a regenerar paisajística y económi- 
$\mathrm{Al}$ amparo de un ambicioso proyecto que se viene desarrollando desde el año 1989, el IBA Emscher Park, y de la Ruta Europea de Patrimonio Industrial, ERIH, el gobierno alemán ha impulsado la recuperación y reutilización de su patrimonio, encabezado por elementos tan notables como el complejo minero de Zollverein, incluido en la lista del Patrimonio Mundial de la UNESCO, favoreciendo el desarrollo económico de la región y regenerando un paisaje desolado por la ruina y el abandono ${ }^{28}$. Precisamente en el marco de

camente el territorio, con la creación del Parque del Emscher. El proyecto contempló tanto su mejora ecológica global como su regeneración económica, contado con cuantiosas inversiones públicas y privadas. En un corredor de unos ochocientos kilómetros cuadrados que discurre entre las ciudades de Duisburg, al Oeste, y Bergkamen, al Este, las instalaciones industriales se han preservado como testimonio de un área cultural cuya seña de identidad ha sido precisamente la industria. En esta línea, se han impulsado diversos proyectos que han permitido introducir nuevos usos en los antiguos contenedores industriales y se han diseñado itinerarios industriales que conectan los lugares que se han recuperado y dan estructura al parque, contando con más de doscientos museos y espacios visitables. El modélico trabajo de catalogación del Patrimonio Industrial que paralelamente se ha impulsado eleva a 3.500 los monumentos industriales con los que cuenta la región, tales como las minas de carbón de Zollverein, incluidas en 2001 en la lista del Patrimonio Mundial de la UNESCO, el gasómetro de Oberhausen o la fábrica Altenberg, también en Oberhausen, por señalar algunos de los más significativos. Véase: U. PETZ, “¿Resucita el ave fénix? La Exposición Internacional de Construcción (Internationale Bauausstellung- IBA) del Parque del Emscher: ideas y resultados de un concepto de planificación orientado hacia la práctica metropolitana", Urban, 11, 2006, pp. 44-69.

${ }^{28}$ La Ruta Europea de Patrimonio Industrial ERIH es una red que enlaza hitos históricos notables del legado de la industrialización europea, los denominados Anchor Points o puntos de referencia. Su principal impulsor, desde el surgimiento de la iniciativa en 1999, fue precisamente el gobierno de Renania del Norte-Westfalia. Se enmarca dentro de las iniciativas que en el ámbito de la Unión Europea se han dirigido a la promoción de los recursos patrimoniales, culturales y naturales, en aras del desarrollo sostenible, como es el caso de las acciones Leader que fomentan las actividades innovadoras en todos los sectores de la economía, a partir de 1991 con Leader I y en adelante con Leader II (19941999) y Leader + (2000-2006). En este mismo período, 2000-2006, se desarrolló la iniciativa INTERREG III del esta iniciativa hemos de inscribir el proyecto Rurh.2010 Capital Europea de la Cultura, dentro de un interesante conjunto de iniciativas que han tenido por objeto la rehabilitación y reutilización de edificios e instalaciones industriales, al amparo de concursos internacionales a los que han concurrido equipos de arquitectos y urbanistas de todo el mundo ${ }^{29}$. En el marco de estas actuaciones se inscribe la implantación de industrias creativas relacionadas con las artes visuales, la música, el cine, el diseño y el teatro, así como el desarrollo de diversos laboratorios de arte público, tales como el Emscherkunts.2013, Urban Arts Ruhr ${ }^{30}$.

Fondo Europeo de Desarrollo Regional, destinada a impulsar el crecimiento equilibrado del territorio europeo, con la cual hemos de relacionar la puesta en marcha de la ERIH. A este respecto puede consultarse: W. EBERT, "ERIH, Ruta Europea de Patrimonio Industrial", en M. A. ALVAREZ (coord.), Estructuras y paisajes industriales, Gijón, 2003, pp. 73-78. Del mismo autor es asimismo destacable la reflexión que lleva a cabo acerca del complejo de Zollverein, en Essen, otrora la mayor mina de carbón de Europa, actualmente considerado como el conjunto minero más bello del mundo, en relación con los proyectos artísticos que en los últimos años se han venido desarrollando: W. EBERT, "El turismo del patrimonio industrial: Zollverein, el complejo minero más bello del mundo", en R. GÓMEZ DE LA IGLESIA. (ed.), Los nuevos centros culturales en Europa, Vitoria-Gasteiz, 2007, pp.121-135.

${ }^{29}$ Entre ellos, especialistas en la arquitectura del paisaje como el alemán Peter Latz, Profesor de la Universidad Técnica de Munich, responsable del diseño y desarrollo del Landschaftspark Duisburg Nord Park. Véase: P. LATZ, "The Idea of Making Time Visible", Topos, 33, 2000, pp. 94-99. Entre los espacios industriales que han sido intervenidos y reutilizados se sitúan el Dortmunder $U$, una fábrica de cerveza que se ha reconvertido en galería de arte y Zeche Nordstern, una antigua mina de carbón que funciona como espacio museístico.

${ }^{30}$ La experiencia de arte público Emscherkunts.2013 fue comisariada por Florian Matzne y contó con la colaboración de Daniel Buren, Ai Weiwei, Mark Dion, Jeppe Hein, Inges Idee, Reiner Maria Matysik, Tobias Rehberger y Tomás Saraceno entre otros artistas. Llevada a cabo entre el 22 de junio y el 6 de octubre de 2013, abarcó una superficie de unos cuarenta y siete kilómetros cuadrados, extendiéndose a lo largo de los núcleos de Duisburg, Dinslaken, Oberhausen, Essen, Bottrop y Gelsenkirchen. Véase: K. P. BUSSE, J. GRÜTJEN \& A. 
Con todo, Fünf Tore funciona como una obra "militante", como una metáfora de la reconstrucción "reparadora", por cuanto genera un entorno poético de reconciliación con la historia. Con ella Hinse actúa, en sintonía con su propia actitud creativa, desde la consciencia del momento presente, desvelando, desenterrando y otorgando de alguna manera una nueva vida al pasado. En atención a los componentes de memoria cultural que la fundamentan, la pieza recalifica el espacio en el que se emplaza, el paisaje urbano $^{31}$, no sólo desde el punto de vista formal, sino también desde el plano conceptual. Aunque el discurso queda abierto a diferentes lecturas e interpretaciones, la razón de ser última de la propuesta no es otra que la de colocar en posición central la identificación con el entorno; un entorno que, en todo caso, se ve reinterpretado y problematizado desde la voluntad de hacer del espectador cómplice y participe del proyecto ${ }^{32}$.

SCHNURR, Emscherkunst 2013. Bildung und Vermittlung im öffentlichen Raum, Oberhausen, 2014.

${ }^{31}$ Acudimos en este caso al concepto "paisaje urbano" tal y como es enunciado por Gordon Cullen al abordar los diferentes papeles que desempeña el ciudadano en los espacios urbanos y la huella que éste imprime: la posesión de los lugares en forma estática y dinámica, la constitución de los diferentes lugares y de sus características propias, los puntos de referencia y sus formas de apropiación por parte de los diferentes grupos sociales. Véase G. CULLEN, El paisaje urbano, Madrid, 1974. Acerca de este aspecto también puede resultar útil la lectura entre otros trabajos de: C. BROTO, Nuevo paisajismo urbano, Barcelona, 2000; J. BORJA, El espacio público, ciudad y ciudadanía, Barcelona, 2003; J. GEHL y L. GEMZOE, Nuevos espacios urbanos, Barcelona, 2002; I. JOSEPH, El transeúnte y el espacio urbano. Ensayo sobre la dispersión del espacio urbano, Barcelona, 2002; E. SOJA, Posmetrópolis. Estudios críticos sobre las ciudades y las regiones, Madrid, 2008.

32 Siguiendo a Javier Maderuelo, la pieza responde a los principales condicionantes de la obra pública en cuanto al mantenimiento de una buena relación de escala con el lugar en el que se ubica, en relación con la consecución de una forma perceptivamente adecuada, en lo que concierne al establecimiento de un diálogo formal y simbólico con el lugar y, por último, al conseguir transmitir un significado. En J. MADERUELO, Caminos de la escultura contemporánea, Salamanca, 2012, pp. 36-37. Acerca del arte público y del arte en el espacio públi-
En este sentido cabe proceder a una lectura del trabajo de Hinse de acuerdo a una óptica política. Siguiendo la estética radical de Jacques Rancière, en particular su reflexión sobre el espectador emancipado, la intervención del creador alemán nos remite a la cuestión del arte en tanto que dispositivo público (político) ${ }^{33}$. De tal modo, se puede considerar que la obra cumple con la misión de despertar la conciencia del espectador y de desarrollar críticamente un conjunto de realidades que afectan a la percepción y a la subjetivación: los usuarios son actores que a partir de la recepción estética, adquieren la posibilidad de ejercer una actuación en el contexto de la acción creativa. Desde la consideración de Rancière la creatividad puede entenderse como una modalidad de resistencia en el espacio político, dentro de los procesos de composición de entramados sociales. La clave descansa en la ruptura con el relativismo estético y la posibilidad de devolver al arte su componente político en el marco de la esfera pública ${ }^{34}$. En este orden

co asimismo, pueden consultarse entre otros trabajos: A. RAVEN, Art in the Public Interest, New York, 1993; W. J. T. MITCHELL, Art and the Public Sphere, Chicago, 1993; M. GOODING, Public Art Space, London, 1998; H. F. SENIE y S. WEBSER, Critical Issues in Public Art: Content, Context and Controversy, Washington D.C., 1998; P. BRANDÄO y A. REMESAR, Espaço público e a Interdisciplinaridade. Espacio público y la interdisciplinaridad, Lisboa, 2000; F. DUQUE, Arte público y espacio político, Madrid, 2001; T. FINKELPEARL, Dialogues in Public Art, Massachussets, 2001; A. REMESAR, Hacia una teoría del arte público, Barcelona, 2007; M. MILES, Cities \& Cultures, London, 2007; M. J. JACOBS, Muerte y vida de las grandes ciudades, Madrid, 2011.

${ }^{33}$ Siguiendo a Jacques Rancière se trataría del punto de igualdad máxima que uniría a creador y espectador, de modo que la participación se convertiría en activa por ambas partes. Así, el espectador se transformaría en participante desde la comunidad y desde la capacidad transformadora fomentada por el talante crítico del arte. De este modo, el espectador emancipado adquiere incluso la capacidad de contra-traducir la narraciones que el artista propone. En J. RANCIÈRE, El espectador emancipado, Buenos Aires, 2010, p. 10.

${ }^{34}$ En palabras de Rancière, "el arte no es político en primer lugar por los mensajes y los sentimientos que transmite sobre el orden del mundo. No es político tampoco por la forma en que representa las estructuras de la 
de cosas, el arte puede ser vivenciado a través de la experiencia estética múltiple, en la que el espectador/usuario se convierte en agente eficiente y componente activo de la práctica artística (Fig. 6).

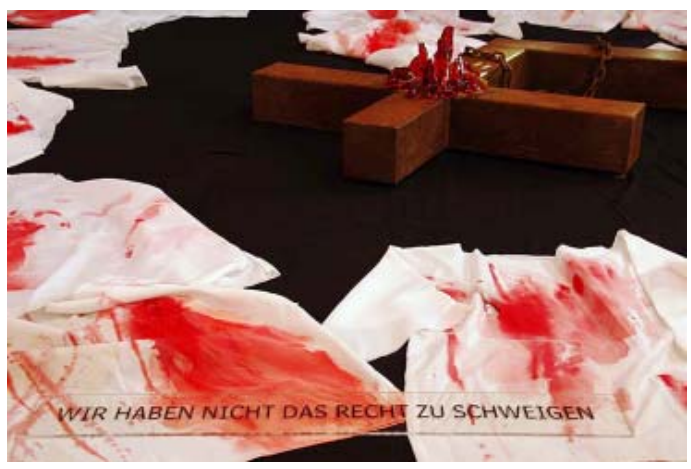

- Fig.6. Leid der Welt. 2011. Ludger Hinse. Catálogo de la exposición Papier trifft Plastik, Kunsthalle Recklinghausen.

En todo caso, no constituye éste el único trabajo realizado por Ludger Hinse con la voluntad de reflexionar sobre la memoria. Por el contrario, desde los años noventa el alemán no ha dejado de inspirarse, al rescate de las huellas del pasado, en temas basados en la espiritualidad, la simbología, la religión, la mitología y la historia, inscritos, bien es cierto, dentro de una temporalidad contemporánea. Adoptando un discurso que no escatima la lectura crítica -particularmente en clave socio-política- una parte de sus creaciones ha respondido a la urgencia de hacer aflorar algunos estigmas propios de la sociedad actual. No obstante, el hermetismo presente en estas obras exige una disponibilidad interpretativa, en cierto modo un acto de complicidad más sofisticado por parte del espectador; en ellas propone la lógica de un análisis, la luz de una razón histórica. En este ámbito resulta especialmente destacable la importancia concedida a la luz, en tanto que ingrediente básico y sustancial de un sector

sociedad, los conflictos o las identidades de los grupos sociales. Es político por la distancia misma que guarda con relación a estas funciones, por el tipo de tiempo y de espacio que establece, por la manera en que divide ese tiempo y prueba ese espacio". En: J. RANCIÈRE, Sobre políticas estéticas, Barcelona, 2005, p. 13. notable de sus propuestas: una luz fluente, expansiva, que en ocasiones funciona como transparencia y que en otras se quiebra, desmaterializando la forma y materializando el espacio ${ }^{35}$. De tal modo, la luz actúa de un modo eficaz, introduciendo dinámicas alegóricas de misterio y ocultación, mostrando la dicotomía existente entre lo que está y lo que no está, entre lo que es y lo que no $\mathrm{es}^{36}$.

Al hilo de estos argumentos, hay una serie de conceptos que, de forma más o menos constante, le han servido como objeto de reflexión, tales como las ideas de soledad, incomunicación, desolación, vacío o alineación. De ellos se hacen eco sus Cabezas Dirigentes que, en clave metafórica -el Confuso, el Místico, el Taciturno, el Preso...- sin apenas apoyaturas exteriores, tratan de la posibilidad de emancipación y de la disposición crítica, superando la decepción y la banalidad. Son obras que enuncian el derecho a la disidencia ${ }^{37}$, abiertas a la duda, al interrogante y a la inestabilidad. Parámetros a los cuales responde asimismo la serie de las Cruces, uno de los proyectos afrontados por Hinse con mayor dedicación en los últimos años, dentro de lo que él mismo ha definido como una cruzada estética ${ }^{38}$. En este caso ha seguido un discurso que, eludiendo toda dimensión visionaria o espiritualista, pone de manifiesto un afán por releer el pasado

35 Véase: M. von VELTHEIM, Zeichen des Lichts, Braunschweig, 2014.

${ }^{36}$ Remite en buena medida al discurso del vacío del hombre desaparecido, siguiendo la reflexión de $\mathrm{M}$. Foucault. A este respecto puede consultarse: M. FOUCAULT, Las palabras y las cosas, Barcelona, 1985, pp.15 y ss.

37 Jesús J. Nebreda trata del derecho a la disidencia "no simplemente como "concesión" graciosa sino como parte integrante de lo racional, de lo verdadero y de lo universal, en tanto que los tres aspectos han de ser contemplados como resultado histórico de sucesivos encuentros con lo "otro" y no como ampliaciones impuestas por el imperialismo de la razón de la fuerza, esto es, de una razón hipostasiada como lo absoluto. En J. J. NEBREDA, Muerte de Dios y Postmodernidad ¿Las largas sombras del Dios muerto?, Granada, 1993, p.181.

${ }^{38}$ Véase: CH. LAMMERT, Weg der Kreuze, Bochum, 2009. 
y someter a examen distintos e incluso contradictorios enfoques de lo sagrado con una voluntad crítica: la experiencia del vacío y de la post-Palabra ${ }^{39}$.

\section{A MODO DE CONCLUSIÓN}

La obra de Ludger Hinse, con todo, en su versatilidad y diversidad plástica, se encuentra estrechamente vinculada a su dimensión vital y a su actividad pública, desarrolladas en el enclave industrial del Ruhr, aspectos que de forma indispensable han de ser considerados para analizar su trabajo en toda su dimensión conceptual y formal.
Buena parte de sus propuestas responden a una visión arqueológica donde la memoria del trabajo y la memoria del lugar ocupan un puesto destacado. Consecuentemente, ha ido elaborando un discurso que ahonda en la interacción arte e industria y, de forma más específica, en la relación entre la escultura y la arqueología industrial. El binomio arte-técnica, por consiguiente, de interés excepcional para comprender un sector importante de la práctica artística contemporánea y del pensamiento teórico gestado a su amparo, se afirma como el principal soporte conceptual sobre el que ha gravitado la labor del creador alemán.

${ }^{39}$ En G. STEINER, Presencias reales. ¿Hay algo en lo que decimos?, Barcelona, 1991, p.118. 